\title{
Morphometric evaluation of 20 Heterorhabditis isolates from Puerto Rico ${ }^{1,2}$
}

\author{
Jessé Roman ${ }^{3}$ and Wilfredo Figueroa
}

\begin{abstract}
From November 1992 through January 1993 soil samples were collected from different localities of Puerto Rico to isolate entomopathogenic nematodes. Samples were baited with the greater wax moth, Galleria mellonella, under laboratory conditions. Nematodes belonging to the genus Heterorhabditis were isolated from 20 of the baited samples. The nematodes could not be properly identified to species but were found closely related to $H$. bacteriophora and $H$. indicus. Thirteen morphometric characters of the infective third-stage juvenile were measured for each isolate and subjected to evaluation by Standard Descriptive Statistics, Stepwise Discriminant Analysis, Canonical Discriminant Analysis and Hierarchical Cluster Analysis. Results suggested the presence of a new species of Heterorhabditis with sufficient variation among the isolates to separate three different subgroups.
\end{abstract}

Key words: Heterorhabditis, morphometrics, nematode, Puerto Rico.

\section{RESUMEN}

Evaluación morfométrica de 20 aislados de Heterorhabditis de Puerto Rico

Entre noviembre de 1992 y enero de 1993 se recogieron muestras de suelo de diferentes localidades de Puerto Rico para aislar nematodos entomopatógenos. Las muestras se inocularon con larvas de la alevilla de los apiarios, Galleria mellonella, bajo condiciones de laboratorio. De 20 de las muestras inoculadas se aisiaron nematodos del género Heterorhabditis. La especie de estos nematodos no se pudo identificar adecuadamente, pero se encontró que estaba relacionada taxonómicamente con $\boldsymbol{H}$. bacteriophora y $H$. indicus. Se midieron trece caracteres morfológicos del tercer estadio juvenil de cada aislado y se sometieron a evaluación por Estadistica Descriptiva Estándar, Análisis Discriminante "Stepwise", Análisis Canónico Discriminante y Análisis "Jerárquico de Grupo". Los resultados sugirieron la presencia de una nueva especie de Heterorhabditis con una marcada variación entre los aislados como para separar tres subgrupos diferentes.

'Received for publication 30 August 1994.

We thank Mrs. C. Chao, Statistics Section, and Mr. M. Antoni, Department of Agricultural Economics and Rural Sociology, Agricultural Experiment Station, University of Puerto Rico, for help in statistical analyses; Dr. Hedwig Hirschmann, Department of Plant Pathology, North Carolina State University, Raleigh, NC, for help in manuscript review; and Dr: G. Poinar, Department of Entomological Sciences, University of California, Berkeley, CA, for review of the morphometrical data.

Professor Emeritus, Department of Crop Protection.

Associate Nematologist, Department of Crop Protection. 


\section{INTRODUCTYON}

In 1975 Poinar erected the family Heterorhabditidae with Heterorhabditis bacteriophora as the type species (Poinar, 1975). At present the family is composed of four species: $H$. bacteriophora Poinar (23 strains); H. zealandica Poinar; H. megidis Poinar, Jackson, and Klein; and $H$. indicus Poinar (Poinar, 1992). The species $H$. heliothidis (=Cromonema heliothidis Khan, Brooks, and Hirschmann) (Khan et al., 1976) was synonymized with $H$. bacteriophora and $H$. zealandica was erected as synonym of a New Zealand population of $H$. heliothidis sensu Wouts by Poinar (Poinar, 1990).

Two keys have been published for the identification of the infective third-stage juveniles of Heterorhabditis. In the first key, the total body length and pharynx length were used to separate the species (Poinar, 1990). The second key included two additional discriminating characters: Ratio $\mathrm{E}$ (distance from head to excretory pore divided by tail length) and Ratio F (body width divided by tail length) (Poinar, 1992).

Keys based on measurements of morphological characters do not always provide a clear discrimination among species. Nevertheless, morphology and morphometrics have been widely used for identification purposes (Hirschmann and Rammah, 1993; Norton and Hinz, 1992; Poinax, 1979; Román and Hirschmann, 1969). In the search for more relevant information that could define taxonomically useful characters to separate species or species groups, taxonomists have subjected morphometrical data to different statistical analyses, such as standard descriptive statistics (SDS), stepwise discriminant analysis (SDA), cluster analysis (CA), and canonical discriminant analysis (CDA). Some of the multivariate tests study the relationship between a group of variables for assessing relative similarities. Different authors have used CDA and hierarchical cluster analysis (HCA) in an effort to divide genera of nematodes into species or taxonomically related groups (Cho and Robbins, 1991; Georgi, 1988; Mota and Eisenback, 1993; Pantone et al.., 1987). Most taxonomists accept that a sound discrimination of species and races can be obtained only when the above tools are used in combination with gel electrophoresis (Akhurst, 1987; Huettle et al., 1983; Hussey, 1979) and DNA fingerprinting (Curran, 1990; Poinar et al., 1987).

The present work was undertaken to determine the species, races or strains of Heterorhabditis present in Puerto Rico and to assess the morphological variation among 20 isolates by subjecting the obtained morphometrical data to SDS, SDA, HCA, and CDA. 


\section{MATERIALS AND METHODS}

Soil samples collected from different localities of Puerto Rico were processed for the isolation of entomopathogenic nematodes. Nematodes obtained were maintained in the laboratory following the methodology described by Figueroa et al. (1993). Twenty of the isolates collected were identified as belonging to the genus Heterorhabditis (Poinar, 1990): Isolates 1 and 2 from Catano, 3 and 20 from Piñones, 4 from Río Piedras, 5 from Loiza, 6 and 7 from Río Grande, 8 from Palmer, 9 to 12 from El Yunque, 13 from Luquillo, 14 and 15 from Ceiba, 16 from Patillas, 17 from Manatí, 18 from Adjuntas and 19 from Villalba. Ensheathed, third-stage juveniles $\left(\mathrm{J}_{3}\right)$ of these isolates were subjected to a morphometric study. The kinds of characters and ratios used were the same as those employed by Poinar (Poinar, 1990; 1992). In addition, the tail cuticle length (distance between tail tip of third-stage juvenile and second-stage cuticle) was included (Table 1).

Ensheathed infective third-stage juveniles were killed by gentle heat and mounted in water on glass slides. Specimens were examined and measured within a six-hour period by using a light microscope with a calibrated camera lucida attachment.

The data were subjected to different statistical analyses. The mean, standard deviation of the mean, and the range were calculated. Also Duncan's Multiple Range Test, SDA, HCA, and CDA were performed with SAS User's Guide (SAS Institute Inc, 1982).

\section{RESULTS}

Standard Descriptive Statistics (SDS):

Table 1 presents morphometrics of 13 characters of 20 isolates of Heterorhabditis. Evaluation of these measurements by SDS revealed their limited use for differentiating the isolates. The majority of the characters overlapped. Only two cases were found with no overlapping; isolates 15 and 19 did not overlap in their greatest width, nor did isolates 18 and 19 in their Ratio $\mathrm{F}$ value.

Mean values of certain characters were found to be statistically significant. Isolate number 9 was significantly longer than all other isolates. Isolate number 19 had a significantly greater body width, Ratio $\mathrm{D}$ and Ratio $\mathrm{F}$ than all other isolates but a significantly smaller Ratio A. Isolate 20 had a significantly greater width and Ratio $F$ value than all the other isolates except isolate 19. The smallest Ratio $\mathrm{F}$ value was found in isolate 18. 
TABLE 1.-Morphometric comparison ( $\mu$ m) of 20 isolates of third-stage juveniles of Heterorhabditis spp.

\begin{tabular}{|c|c|c|c|c|c|}
\hline \multirow[b]{2}{*}{ Character } & \multicolumn{5}{|c|}{ Population number $(N=25)$} \\
\hline & 1 & 2 & 3 & 4 & 5 \\
\hline Body Length & $\begin{array}{l}537 \pm 21.14 \mathrm{i} \\
(495-571)\end{array}$ & $\begin{array}{l}560 \pm 15.75 \mathrm{cdefgh} \\
(529-590)\end{array}$ & $\begin{array}{l}550 \pm 33.40 \mathrm{ghi} \\
(490-605)\end{array}$ & $\begin{array}{l}543 \pm 24.43 \mathrm{hi} \\
(495-586)\end{array}$ & $\begin{array}{l}550 \pm 18.15 \mathrm{ghi} \\
(510-586)\end{array}$ \\
\hline Greatest Width & $\begin{array}{l}20 \pm 1.2 \text { fgh } \\
(18-23)\end{array}$ & $\begin{array}{l}20 \pm 0.82 \mathrm{fgh} \\
(19-22)\end{array}$ & $\begin{array}{l}19 \pm 1.22 \mathrm{ghij} \\
(18-23)\end{array}$ & $\begin{array}{l}20 \pm 1.31 \text { fghi } \\
(18-24)\end{array}$ & $\begin{array}{l}20=1.45 \mathrm{efg} \\
(10-24)\end{array}$ \\
\hline $\begin{array}{l}\text { Dist. Head } \\
\text { to Ex. Pore }\end{array}$ & $\begin{array}{l}93 \pm 5.54 g \\
(81-102)\end{array}$ & $\begin{array}{l}95 \pm 3.39 \mathrm{efg} \\
(88-100)\end{array}$ & $\begin{array}{l}98 \pm 6.57 \text { bcdef } \\
(86-112)\end{array}$ & $\begin{array}{l}96 \pm 5.37 \mathrm{defg} \\
(88-110)\end{array}$ & $\begin{array}{l}95 \div 3.81 \mathrm{efg} \\
(86-102)\end{array}$ \\
\hline $\begin{array}{l}\text { Dist. Head } \\
\text { to Nerve Ring }\end{array}$ & $\begin{array}{l}80=4.67 \mathrm{fg} \\
(69-90)\end{array}$ & $\begin{array}{l}80 \pm 3.74 \text { efg } \\
(71-88)\end{array}$ & $\begin{array}{l}80=5.45 f g \\
(68-98)\end{array}$ & $\begin{array}{l}81 \pm 591 \text { defg } \\
(69-93)\end{array}$ & $\begin{array}{l}81 \cdot 3.57 \text { cdefg } \\
(74-90)\end{array}$ \\
\hline $\begin{array}{l}\text { Dist. Head to } \\
\text { Pharynx base }\end{array}$ & $\begin{array}{l}111 \pm 5.83 \mathrm{cdef} \\
(100-119)\end{array}$ & $\begin{array}{l}110 \pm 5.50 \mathrm{ef} \\
(100-119)\end{array}$ & $\begin{array}{l}111 \pm 6.79 \mathrm{def} \\
(98-124)\end{array}$ & $\begin{array}{l}113 \pm 3.99 b c d e f \\
(105-119)\end{array}$ & $\begin{array}{l}112 \pm 5.38 \text { bcdef } \\
(98-119)\end{array}$ \\
\hline Total Tail Length & $\begin{array}{l}94 \pm 8.41 \text { lahi } \\
(62-105)\end{array}$ & $\begin{array}{l}99 \pm 3.73 b c d \\
(92-106)\end{array}$ & $\begin{array}{l}94 \pm 3.97 \mathrm{ghi} \\
(86-103)\end{array}$ & $\begin{array}{l}90 \pm 4.43 j \\
(78-96)\end{array}$ & $\begin{array}{l}95 \pm 6.97 \mathrm{fghi} \\
(84 \cdot 112)\end{array}$ \\
\hline Tail Cuticle Length & $\begin{array}{l}33 \pm 3.80 \text { defg } \\
(24-40)\end{array}$ & $\begin{array}{l}36 \pm 2.59 b c d \\
(30-41)\end{array}$ & $\begin{array}{l}33 \pm 3.5 \text { lefgh } \\
(26-40)\end{array}$ & $\begin{array}{l}29 \pm 3.22 \mathrm{i} \\
(24-36)\end{array}$ & $\begin{array}{l}32 \pm 5.17 \mathrm{fgh} \\
(24-42)\end{array}$ \\
\hline Ratio $A^{2}$ & $\begin{array}{l}27 \pm 1.51 \text { def } \\
(24-30)\end{array}$ & $\begin{array}{l}28=1.26 \mathrm{abc} \\
(26-31)\end{array}$ & $\begin{array}{l}28=1.69 a b c \\
26-33)\end{array}$ & $\begin{array}{l}28 \pm 1.60 \mathrm{~cd} \\
(24-30)\end{array}$ & $\begin{array}{l}27 \pm 1.46 \mathrm{ed} \\
(24-31)\end{array}$ \\
\hline Ratio $B^{2}$ & $\begin{array}{l}4.8 \pm 0.23 \mathrm{cdef} \\
(4.4-5.3)\end{array}$ & $\begin{array}{l}5.1 \leq 0.30 a b \\
(4.8-5.7)\end{array}$ & $\begin{array}{l}5.0 \pm 0.23 \mathrm{abcde} \\
(4.6-5.4)\end{array}$ & $\begin{array}{l}4.8=0.22 \mathrm{de}= \\
(4.4-5.2)\end{array}$ & $\begin{array}{l}4.9 \pm 0.31 \text { hcdef } \\
(4.5-5.8)\end{array}$ \\
\hline Ratio C: & $\begin{array}{l}5.9 \div 0.82 \text { abcdefg } \\
(5.2-8.6)\end{array}$ & $\begin{array}{l}5.7 \pm 0.19 g h i \\
(5.4-6.1)\end{array}$ & $\begin{array}{l}5.8 \pm 0.29 b c d e f g \\
(5.4-6.6)\end{array}$ & $\begin{array}{l}6.0 \pm 0.20 a b c \\
(5.6-6.5)\end{array}$ & $\begin{array}{l}5.8 \pm 0.39 b c d e f g \\
(5.0-6.5)\end{array}$ \\
\hline Ratio D & $\begin{array}{l}0.84 \pm 0.04 \mathrm{fgh} \\
(0.77-0.92)\end{array}$ & $\begin{array}{l}0.86 \pm 0.03 b c d e f \\
(0.78-0.93)\end{array}$ & $\begin{array}{l}0.88 \pm 0.03 b \\
(0.82-0.94)\end{array}$ & $\begin{array}{l}0.85 \pm 0.04 \mathrm{cdef} g \\
(0.80-0.94)\end{array}$ & $\begin{array}{l}0.85 \pm 0.03 \text { defgh } \\
(0.77-0.90)\end{array}$ \\
\hline Ratio E & $\begin{array}{l}1.02 \pm 0.14 \mathrm{de} \\
(0.86-1.42)\end{array}$ & $\begin{array}{l}0.96 \pm 0.05 \mathrm{fgh} \\
(0.88 \cdot 1.09)\end{array}$ & $\begin{array}{l}1.04 \pm 0.07 \mathrm{abcd} \\
(0.93-1.21)\end{array}$ & $\begin{array}{c}1.07 \pm 0.07 \mathrm{ab} \\
10.94-1.28)\end{array}$ & $\begin{array}{l}1.01 \pm 0.09 \mathrm{def} \\
(0.85-1.17)\end{array}$ \\
\hline Ratio Fi & $\begin{array}{l}0.22 \pm 0.03 \mathrm{~cd} \\
(0.19-0.32)\end{array}$ & $\begin{array}{c}0.20 \pm 0.01 \mathrm{~h} \\
(0.18-0.23)\end{array}$ & $\begin{array}{c}0.21 \pm 0.01 \text { efgh } \\
(0.18-0.24)\end{array}$ & $\begin{array}{l}0.22 \pm 0.01 \mathrm{~cd} \\
(0.20-0.26)\end{array}$ & $\begin{array}{l}0.21 \pm 0.02 \mathrm{cdefg} \\
(0.18-0.26)\end{array}$ \\
\hline
\end{tabular}

Values are Means. \pm S.D., (Range). Means followed by the same lctter within a row are not different according to the Duncan's Multiple Range Test $(P=0.05$ ) 'Body length'Greatest width. 'Body length/Dist. head to pharynx base. "Body length/Total tail length. "Dist. head to Ex. pore/Dist. head to pharynx base. "Dist. head to Ex. pore/Total tail length. Greatest width/Total tail length. 
TABLE 1.-Morphometric comparison (um) of 20 isolates of third-stage juveniles of Heterorhahditis spp. (Cont.)

\begin{tabular}{|c|c|c|c|c|c|}
\hline \multirow[b]{2}{*}{ Character } & \multicolumn{5}{|c|}{ Population number $(\mathrm{N}=2.5 \mathrm{i}$} \\
\hline & 6 & 7 & 8 & 9 & 10 \\
\hline Body Length & $\begin{array}{l}569 \pm 23.57 b c d e \\
(524-610\}\end{array}$ & $\begin{array}{l}568 \pm 34.92 \text { bcdef } \\
(495-638)\end{array}$ & $\begin{array}{l}560 \pm 21.38 \text { cdefgh } \\
(529 \cdot 605)\end{array}$ & $\begin{array}{l}595 \pm 32.95 a \\
(514-662)\end{array}$ & $\begin{array}{l}553 \pm 19,42 \text { efghi } \\
(514-586)\end{array}$ \\
\hline Greatest Width & $\begin{array}{l}21 \pm 1.53 \mathrm{de} \\
(19-24)\end{array}$ & $\begin{array}{l}21 \pm 1.58 \mathrm{ef} \\
(18-24)\end{array}$ & $\begin{array}{l}20 \pm 1.08 \text { ef } \\
(19-23)\end{array}$ & $\begin{array}{l}22 \pm 2.16 c \\
(19-26)\end{array}$ & $\begin{array}{l}18 \pm 0.87 h i j \\
(18-22)\end{array}$ \\
\hline $\begin{array}{l}\text { Dist. Head } \\
\text { to Ex. Pore }\end{array}$ & $\begin{array}{l}98 \pm 7.22 b c d e f \\
(83-117)\end{array}$ & $\begin{array}{l}98=4.20 b c d e \\
(93-1.07)\end{array}$ & $\begin{array}{l}95 \pm 3.95 f_{\mathrm{B}} \\
(88-102)\end{array}$ & $\begin{array}{l}101 \cdot 5.64 a b \\
\left(93-117^{\prime}\right.\end{array}$ & $\begin{array}{l}96 \pm 3.78 \text { def } \\
(90-105)\end{array}$ \\
\hline $\begin{array}{l}\text { Dist. Head } \\
\text { to Nerve Ring }\end{array}$ & $\begin{array}{l}86 \pm 6.12 a \\
(74-102)\end{array}$ & $\begin{array}{l}82 \pm 4.67 \text { bcdef } \\
(76-93)\end{array}$ & $\begin{array}{l}82 \pm 3.35 \text { bedefg } \\
(71-88)\end{array}$ & $\begin{array}{l}84 \pm 3.15 \mathrm{Jabc} \\
(79-90)\end{array}$ & $\begin{array}{l}80 \pm 3.29 \mathrm{efg} \\
(74-86)\end{array}$ \\
\hline $\begin{array}{l}\text { Dist. Head to } \\
\text { Pharynx base }\end{array}$ & $\begin{array}{l}113 \pm 9.17 \text { bcdef } \\
(83-131)\end{array}$ & $\begin{array}{l}115 \pm 5.45 a b c \\
(102-126)\end{array}$ & $\begin{array}{l}110 \pm 5.38 f \\
(100-121)\end{array}$ & $\begin{array}{l}117 \pm 4.69 \mathrm{a} \\
(107-125)\end{array}$ & $\begin{array}{l}114 \pm 3.24 a b c d \\
(110-121)\end{array}$ \\
\hline Total Tail Length & $\begin{array}{l}103 \pm 5.37 a \\
(94-118)\end{array}$ & $\begin{array}{l}99 \pm 4.85 \mathrm{~cd} \\
(88-106)\end{array}$ & $\begin{array}{l}102 \pm 5.64 a b \\
(94-115)\end{array}$ & $\begin{array}{l}101 \pm 5.44 a b c \\
(92-116)\end{array}$ & $\begin{array}{l}93 \div 3.48 i j \\
(84-98)\end{array}$ \\
\hline Tail Cuticte Length & $\begin{array}{l}39 \pm 4.36 a \\
(30 \cdot 48)\end{array}$ & $\begin{array}{l}35 \pm 3.54 \mathrm{cdef} \\
(2 \delta-12)\end{array}$ & $\begin{array}{l}38 \pm 5.24 a b \\
(30-52)\end{array}$ & $\begin{array}{l}37 \pm 5.18 a b c \\
(30-48)\end{array}$ & $\begin{array}{l}31 \pm 3.03 \mathrm{hi} \\
(24-36)\end{array}$ \\
\hline Ratio $A^{\prime}$ & $\begin{array}{l}27 \pm 1.51 \text { def } \\
(25-30)\end{array}$ & $\begin{array}{l}28: 1.89 b c d \\
(24-31)\end{array}$ & $\begin{array}{l}28 \pm 1.71 \mathrm{~cd} \\
(24-30)\end{array}$ & $\begin{array}{l}27 \pm 1.89 \text { def } \\
(25-31)\end{array}$ & $\begin{array}{l}29 \pm 1.41 \mathrm{ab} \\
(25-31)\end{array}$ \\
\hline Ratio B: & $\begin{array}{l}5.1 \pm 0.52 \mathrm{ab} \\
(4.3-7.2)\end{array}$ & $\begin{array}{l}4.9 \pm 0.30 \text { abcde } \\
(4.4-5.7)\end{array}$ & $\begin{array}{l}5.1 \pm 0.25 \mathrm{ab} \\
(4.6-5.5)\end{array}$ & $\begin{array}{l}5.1 \pm 0.29 \mathrm{a} \\
(4.3-5.8)\end{array}$ & $\begin{array}{l}4.8 \pm 0.17 \mathrm{cdef} \\
(4.6-5.1)\end{array}$ \\
\hline Ratio C & $\begin{array}{l}5.5 \pm 0.30 \text { hij } \\
(5.1-6.1)\end{array}$ & $\begin{array}{l}5.8 \pm 0.29 \mathrm{defg} \\
\{5.3-6.3 !\end{array}$ & $\begin{array}{l}5.5 \pm 0.32 \mathrm{ij} \\
4.9-6.1)\end{array}$ & $\begin{array}{l}5.9 \pm 0.44 a b c d e f g \\
(5.2-6.6)\end{array}$ & $\begin{array}{l}6.0 \pm 0.26 \mathrm{abca} \\
(5.5 \cdot 6.5)\end{array}$ \\
\hline Ratio $D^{6}$ & $\begin{array}{l}0.87 \pm 0.07 b c d \\
(0.77-1.18)\end{array}$ & $\begin{array}{l}0.86 \pm 0.03 \text { cdefg } \\
(0.80-0.93)\end{array}$ & $\begin{array}{l}0.86 \pm 0.03 b c d e \\
(0.81-0.95)\end{array}$ & $\begin{array}{l}0.84 \pm 0.06 \mathrm{efgh} \\
(0.71-0.94)\end{array}$ & $\begin{array}{l}0.84 \pm 0.03 \text { defgh } \\
(0.80-0.89)\end{array}$ \\
\hline Ratio $\mathrm{E}^{\mathbf{3}}$ & $\begin{array}{l}0.95 \pm 0.08 \mathrm{gh} \\
(0.81-1.17)\end{array}$ & $\begin{array}{l}1.00 \pm 0.04 \mathrm{defg} \\
(0.91-1.08)\end{array}$ & $\begin{array}{l}0.94 \pm 0.06 h \\
(0.79-1.06)\end{array}$ & $\begin{array}{l}0.97 \pm 0.08 \text { fgh } \\
(0.86-1.19)\end{array}$ & $\begin{array}{l}1.04 \pm 0.04 \mathrm{abcd} \\
(0.97 \cdot 1.11)\end{array}$ \\
\hline Ratio Fn & $\begin{array}{l}0.20 \pm 0.02 \mathrm{fgh} \\
(0.18-0.24)\end{array}$ & $\begin{array}{l}0.21 \pm 0.02 \text { defgh } \\
(0.18-0.25)\end{array}$ & $\begin{array}{l}0.20 \pm 0.01 \mathrm{~h} \\
(0.17-0.23)\end{array}$ & $\begin{array}{l}0.22 \pm 0.02 \mathrm{cde} \\
(0.18-0.25)\end{array}$ & $\begin{array}{l}0.21=0.01 \mathrm{efgh} \\
(0.19 \cdot 0.24)\end{array}$ \\
\hline
\end{tabular}

Values are Means, \pm S.D., (Range). Means followed by the same letter within a row are not different according to the Duncan's Multiple Range Test $(P=0.05$.

'Body length/Greatest width. "Body length/Dist. head to pharynx base. Body length/Total tail length. "Dist. hond to Ex. pore/Dist. head to pharynx base. Dist. head to Ex. pore/Total tail length. "Greatest width/Lotal tail length. 
TABLE 1.-Morphometric comparison ( $u$ m) of 20 isolates of third-stage juveniles of Heterorhabditis spp. (Cont.)

\begin{tabular}{|c|c|c|c|c|c|}
\hline \multirow[b]{2}{*}{ Character } & \multicolumn{5}{|c|}{ Population number $(\mathrm{N}=2 \tilde{5})$} \\
\hline & 11 & 12 & 13 & 14 & 15 \\
\hline Bady Lengill & $\begin{array}{l}567 \pm 26.22 b c d e f g \\
(529-629)\end{array}$ & $\begin{array}{l}572 \pm 22.59 \mathrm{bcd} \\
(519-619)\end{array}$ & $\begin{array}{l}546 \pm 34.38 \mathrm{hi} \\
(476-600)\end{array}$ & $\begin{array}{l}555 \div 28.38 \text { defghi } \\
(500-610)\end{array}$ & $\begin{array}{l}544 \pm 28.90 \mathrm{hi} \\
(486 \cdot 581)\end{array}$ \\
\hline Greates: Width & $\begin{array}{l}19 \pm 0.86 g h i j \\
(1.8-22)\end{array}$ & $\begin{array}{l}21 \pm 1.71 \mathrm{de} \\
(19-24)\end{array}$ & $\begin{array}{l}20 \pm 1.78 \mathrm{ef} \\
(18-23)\end{array}$ & $\begin{array}{l}19 \pm 0.81 \text { ghij } \\
(18-22)\end{array}$ & $\begin{array}{l}19 \pm 0.65 j \\
(18-20)\end{array}$ \\
\hline $\begin{array}{l}\text { Dist. Head } \\
\text { to Ex. Pore }\end{array}$ & $\begin{array}{l}97 \pm 3.50 \mathrm{cdef} \\
(90-105)\end{array}$ & $\begin{array}{l}100 \pm 3.99 a b c \\
(93-107)\end{array}$ & $\begin{array}{l}98 \pm 6.68 \mathrm{bcdef} \\
(86-112)\end{array}$ & $\begin{array}{l}97 \pm 4.60 \text { def } \\
(86-105)\end{array}$ & $\begin{array}{l}97 \pm 3.92 \mathrm{cdef} \\
(88-105)\end{array}$ \\
\hline $\begin{array}{l}\text { Dist. Head } \\
\text { to Nerve Ring }\end{array}$ & $\begin{array}{l}81 \pm 2.89 \mathrm{defg} \\
(74-88)\end{array}$ & $\begin{array}{l}83 \pm 3.89 b c d e \\
(74-90)\end{array}$ & $\begin{array}{l}83 \pm 5.80 \mathrm{bcde} \\
(74-98)\end{array}$ & $\begin{array}{l}83 \pm 4.28 a b c d \\
(71-90)\end{array}$ & $\begin{array}{l}82 \pm 3.63 b c d e f g \\
(71-88)\end{array}$ \\
\hline $\begin{array}{l}\text { Dist. Head to } \\
\text { Pharynx base }\end{array}$ & $\begin{array}{l}115 \pm 3.62 a b c \\
(107-121)\end{array}$ & $\begin{array}{l}114 \pm 3.23 a b c d e \\
(107-119)\end{array}$ & $\begin{array}{l}112 \pm 6.89 \mathrm{cdef} \\
(95-124)\end{array}$ & $\begin{array}{l}112=5.37 \mathrm{cdef} \\
(95-119)\end{array}$ & $\begin{array}{l}113 \pm 3.56 \mathrm{bcdef} \\
(105 \cdot 119)\end{array}$ \\
\hline Total Tail Length & $\begin{array}{l}94 \pm 4.45 \mathrm{hi} \\
(86-104)\end{array}$ & $\begin{array}{l}98 \pm 3.15 \mathrm{de} \\
(90-104)\end{array}$ & $\begin{array}{l}95 \pm 5.74 \text { efghi } \\
(84-104)\end{array}$ & $\begin{array}{l}97 \pm 5.33 \text { defg } \\
(90-109)\end{array}$ & $\begin{array}{l}90 \pm 5.06 j \\
(78-98)\end{array}$ \\
\hline Tail Cuticle Length & $\begin{array}{l}32 \pm 4.18 g h \\
(24-42)\end{array}$ & $\begin{array}{l}34 \pm 3.06 \text { defg } \\
(30-41)\end{array}$ & $\begin{array}{l}32 \pm 4.22 \mathrm{fgh} \\
(23-40)\end{array}$ & $\begin{array}{l}34 \pm 3.62 \text { cdefg } \\
(28-43)\end{array}$ & $\begin{array}{l}29 \pm 3.47 \mathrm{j} \\
(24-36)\end{array}$ \\
\hline Ratio A: & $\begin{array}{l}29 \pm 1.46 a \\
(25-33)\end{array}$ & $\begin{array}{l}27 \pm 1.85 \text { de } \\
(24-31)\end{array}$ & $\begin{array}{l}26 \pm 1.56 e f \\
(23-29)\end{array}$ & $\begin{array}{l}29 \pm 1.42 \mathrm{ab} \\
(26-31)\end{array}$ & $\begin{array}{l}29 \pm 1.35 \mathrm{a} \\
(26-31)\end{array}$ \\
\hline Ratio $B^{:}$ & $\begin{array}{l}5.0 \pm 0.20 \text { abcde } \\
(4.7-5.3)\end{array}$ & $\begin{array}{l}5.0 \pm 0.18 \mathrm{abcd} \\
(4.7-5.4)\end{array}$ & $\begin{array}{l}5.0 \pm 0.37 b c d e f \\
(4.2-6.0)\end{array}$ & $\begin{array}{l}5.0=0.25 a b c d e \\
(4.4-5.4)\end{array}$ & $\begin{array}{l}4.8 \pm 0.21 \mathrm{ef} \\
(4.3-\tilde{0} .3)\end{array}$ \\
\hline Ratio C & $\begin{array}{l}6.0 \pm 0.25 a b \\
(5.4-6.5)\end{array}$ & $\begin{array}{l}5.8 \pm 0.22 b c d e f g \\
(5.4-6.4)\end{array}$ & $\begin{array}{l}5.8 \pm 0.30 \mathrm{efg} \\
(5.0-6.5)\end{array}$ & $\begin{array}{l}5.7 \pm 0.27 \text { fghi } \\
(5.2-6.1)\end{array}$ & $\begin{array}{l}6.1 \pm 0.24 a \\
(5.6-6.8)\end{array}$ \\
\hline Ratio D' & $\begin{array}{l}0.85 \pm 0.03 \text { defgh } \\
(0.80-0.92)\end{array}$ & $\begin{array}{l}0.88 \pm 0.04 b \\
\{0.80-1.00\}\end{array}$ & $\begin{array}{l}0.88 \pm 0.03 b c \\
(0.80-0.94)\end{array}$ & $\begin{array}{l}0.86 \pm 0.04 \mathrm{bcd} \\
10.79-0.94 !\end{array}$ & $\begin{array}{l}0.86 \pm 0.03 \mathrm{cdefg} \\
(0.77-0.89)\end{array}$ \\
\hline Ratio $\mathrm{E}^{\circ}$ & $\begin{array}{l}1.04 \pm 0.05 a b c d \\
(0.92-1.14)\end{array}$ & $\begin{array}{l}1.02 \pm 0.04 \mathrm{cde} \\
(0.94-1.11)\end{array}$ & $\begin{array}{l}1.03 \pm 0.07 \mathrm{bcd} \\
(0.90-1.22)\end{array}$ & $\begin{array}{l}1.00 \pm 0.06 \mathrm{defg} \\
(0.88-1.13 .)\end{array}$ & $\begin{array}{l}1.08 \pm 0.07 \mathrm{a} \\
(0.90-1.22)\end{array}$ \\
\hline Rutio Fi & $\begin{array}{l}0.21 \pm 0.0 \text { lefgh } \\
(0.18-0.23)\end{array}$ & $\begin{array}{l}0.22 \pm 0.02 \mathrm{cdef} \\
(0.19-0.25)\end{array}$ & $\begin{array}{l}0.22 \pm 0.02 \text { cdefg } \\
(0.18-0.26)\end{array}$ & $\begin{array}{c}0.20 \pm 0.01 h \\
(0.18-0.24)\end{array}$ & $\begin{array}{l}0.21 \pm 0.01 \text { defyh } \\
(0.19-0.24)\end{array}$ \\
\hline
\end{tabular}

Values are Means, $\neq$ S.D., (Range). Means followed by the same letter within a row are not different according to the Duncan's Multiple Range Test. ( $P=0.05$ ).

Body length/Greatcst width. "Body length/Dist. head to pharynx base. "Body length/Total tail length. "Dist. head to Ex. pore/Dist. head to pharynx base. Dist. head to Ex. pore/Total tail length. "Greatest width/Total tail longth. 
TABLE 1.-Morphometric comparison ( $\mu$ m) of 20 isolates of third-stage juveniles of Heterorhabditis spp. (Cont.)

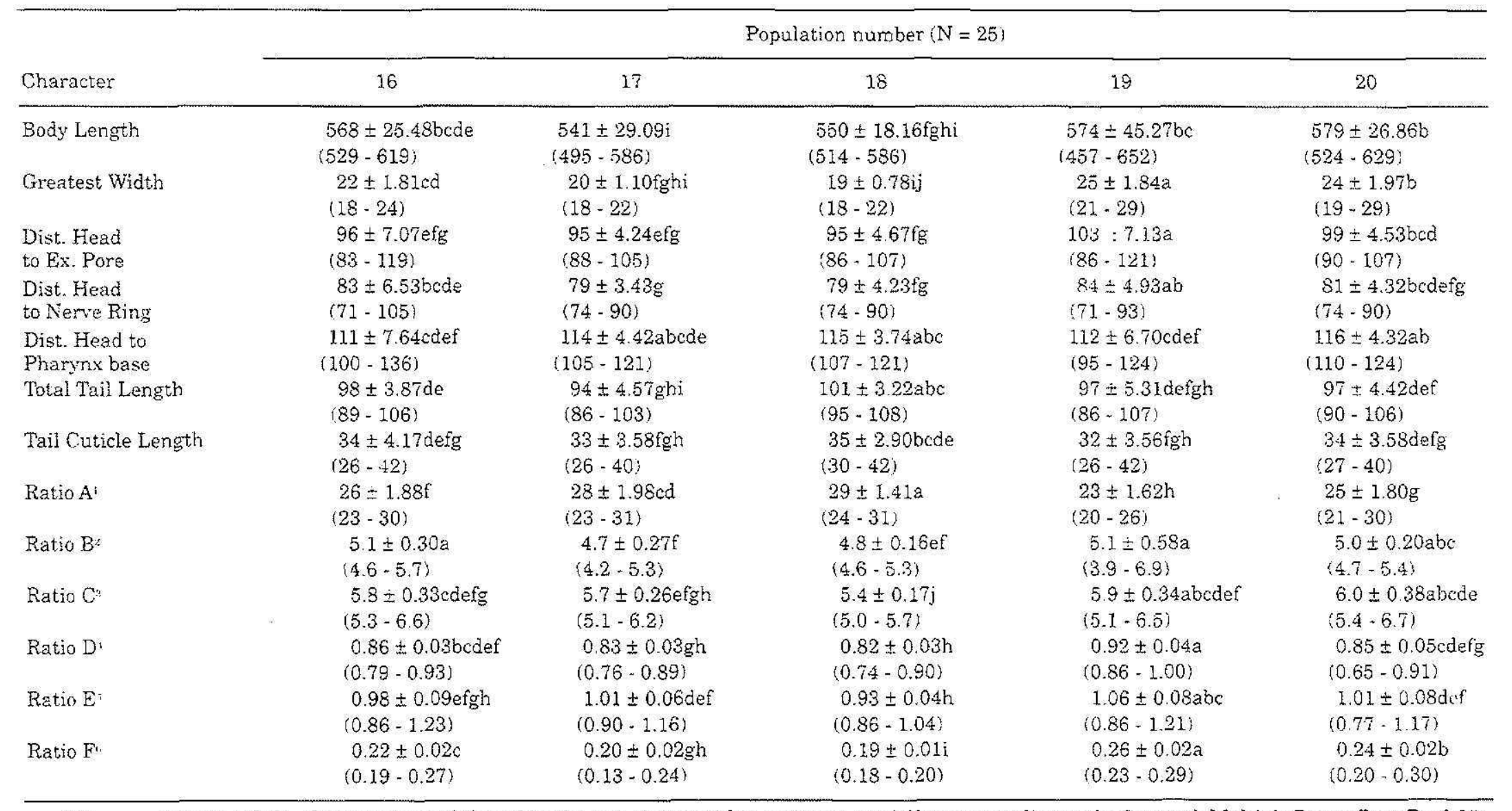

Values are Means, \pm S.D., (Range). Means followed by the same letter within a row are not different according to the $D$ uncan's $M$ iltiple Range Test $(P=0.05$ ).

Body lenoth/Greatest width. ${ }^{2 B o d y}$ length/Dist. head to pharynx base. "Body length/Total tail length. Dist. head to Ex. pore'Dist. head to pharynx base. Dist. head to Ex. pore/Total tail length. "Greatest width/Total tail length. 
Stepwise Discriminant Analysis (SDA):

The 13 morphometric characters were entered in the SDA (forward and backward selection methods) to determine the variables or diagnostic characters that accounted for most of the variation of the data. The forward version of the SDA selected 10 variables; the backward, 12. For this study the four variables with the highest Eigenvalues and the highest correlation in decreasing significance were selected for further analyses (Tables 2 and 3). These correlations were significant at $\mathrm{P}$ $=0.01$. The canonical variables constituted 1 ) body length, 2 ) greatest width, 3) distance from head to pharynx base, and 4) tail length. The first and second canonical variables accounted for $88 \%$ of the total variance (Table 2). The third and fourth added little to the variance in the data. Nevertheless, they were selected because of their importance in the taxonomy of the genus.

Hierarchical Cluster Analysis (HCA):

Input variables for HCA were the same four variables as obtained by SDA. Three subgroups were indicated in the dendrogram according to the HCA (Figure 1). Subgroup 1 included 10 isolates in three divisions. Isolates $1,3,5,13$, and 14 formed the first division. Isolates 15 , 4,17 , and 10 formed the second division, which was clustered with the first. Isolate 11 composed the third division clustered with the former two divisions. Subgroup 2 included seven isolates in two divisions. Isolates 2,8 , and 16 formed the first division clustered with isolates 6,7 , 12 , and 18 in the second division. Subgroup 2 was clustered with subgroup 1. Subgroup 3 included isolates 9,20 and 19 clustered with the other two subgroups.

Canonical Discriminant Analysis (CDA):

Figures 2 through 5 present scatterplots of canonical means of 20 isolates of Heterorhabditis.

TABLE 2.- Eigenvalues of the correlation matrix generated during Stepwise Discriminant Analysis of Heterorhabditis isolates.

\begin{tabular}{lcccc}
\hline $\begin{array}{l}\text { Canonical } \\
\text { Variable' }\end{array}$ & Eigenvalue & Difference & Proportion & Cumulative \\
\hline C 1 & 1.28 & 0.76 & 0.62 & 0.62 \\
C 2 & 0.53 & 0.36 & 0.26 & 0.88 \\
C 3 & 0.17 & 0.09 & 0.08 & 0.96 \\
C 4 & 0.08 & - & 0.04 & 1.00 \\
\hline
\end{tabular}

${ }^{\prime} \mathrm{C} 1$ = Total length, $\mathrm{C} 2$ = Greatest width, C $3=$ Distance from head to pharynx base, C 4 = Tail length. 
J. Agric, Univ. P.R. vOL. 79, NO. 1-2, JANUARY/APRLL 1995

TABLE 3.-Correlation coefficients among the variables chosen by Stepuis Discriminant. Analysis.

\begin{tabular}{lccc}
\hline & $\mathrm{TL}^{\mathrm{i}}$ & $\mathrm{GW}$ & $\mathrm{DHPh}$ \\
\hline $\mathrm{GW}$ & 0.5403 & & \\
$\mathrm{D} \mathrm{H} \mathrm{Ph}$ & 0.3471 & 0.1169 & \\
$\mathrm{~T}$ & 0.4789 & 0.2703 & 0.0897 \\
\hline
\end{tabular}

'T $L=$ Total length, $G W=$ Greatest width, $D H P h=$ Distance from head to pharynx base, T- Tail length.

Input variables for CDA were the same four variables as obtained by SDA.

When the means of the first and second canonical variables were plotted on canonical axes, and borderlines following results of HCA traced, the three principal subgroups of isolates observed in the HCA were delimited (Figure 2). Subgroup 1 was composed of 10 isolates ( 1 , $3,4,5,10,11,13,14,15$, and 17). Subgroup 2 comprised six isolates ( 2 , $6,7,8,12$, and 16) and subgroup 3 comprised three isolates $(9,19$, and $20)$.

Similarly, when means of the first and fourth canonical variables (Figure 3), second and fourth variables (Figure 4) and second and third variables (Figure 5) were plotted on canonical axes, the same three subgroups were delimited. Isolate 18 was found encompassed within

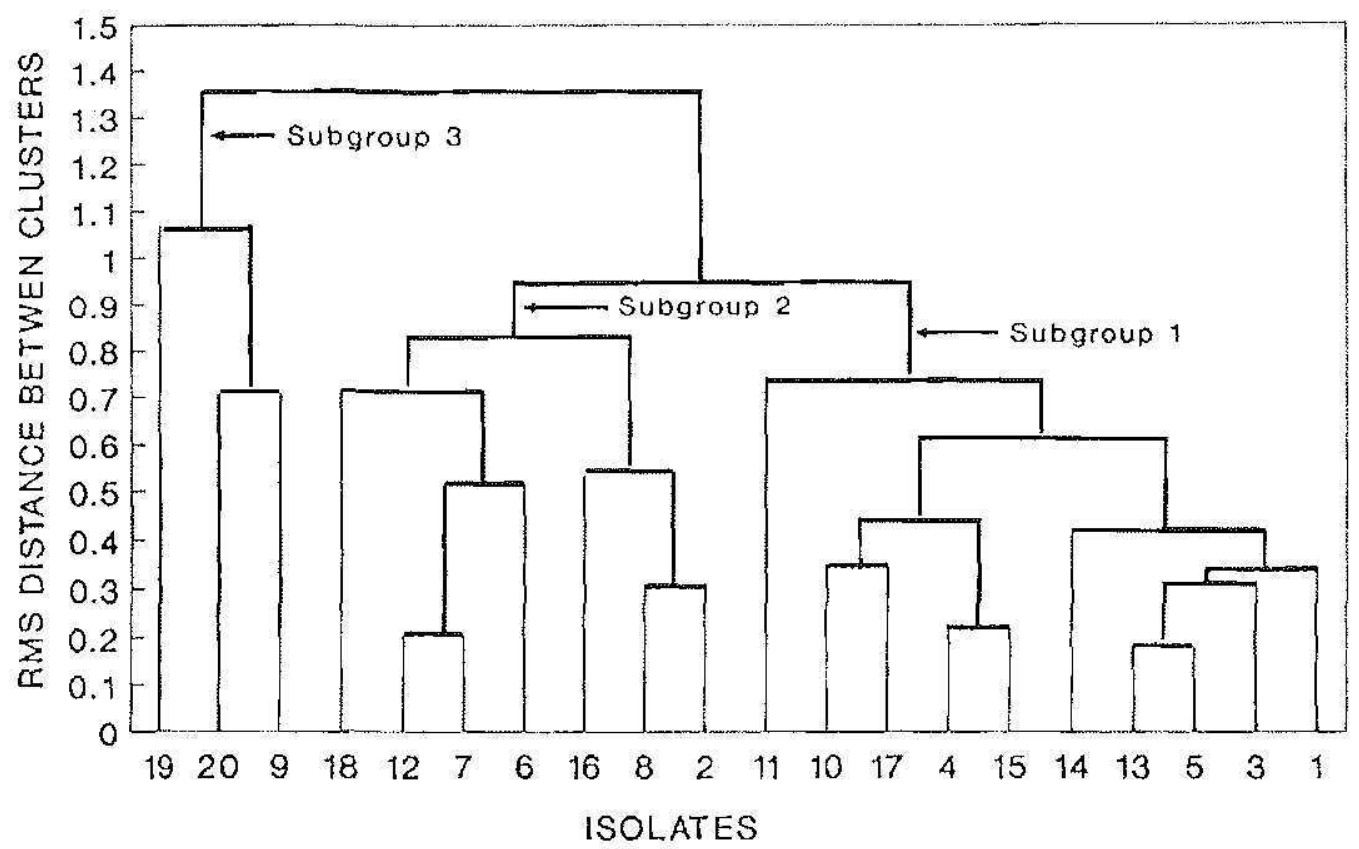

FuUne 1. Dendrogram showing the clustering of 20 Puerto Rican isolates of Heterorhabditis based on mean values of four morphometric variables of $\mathrm{J}_{3}$. 
subgroup 2, when the means of the first and fourth canonical variables and of the second and fourth variables were plotted on canonical axes (Figures 3 and 4). Otherwise, this isolate was observed away from subgroup 2 (Figures 2 and 5 ).

\section{DISCUSSION}

The morphometric data obtained in this study indicate that the 20 isolates of Heterorhabditis collected from Puerto Rico belong to a new species closely related to $H$. bacteriophora and $H$. indicus. ${ }^{5}$ In spite of the fact that morphometrics supported the existence of a new species, the variability in dimensions among the 20 isolates was great, and much overlapping occurred within each character. The SDS separated isolates $9,18,19$, and 20 as significantly different from each other on the basis of certain morphometric characters (Table 1). Results of the HCA (Figure 1) and CDA (Figures 2 through 5) support these observations.

The forward and backward versions of the SDA selected 10 and 12 variables, respectively. Such a large number of variables, when used in

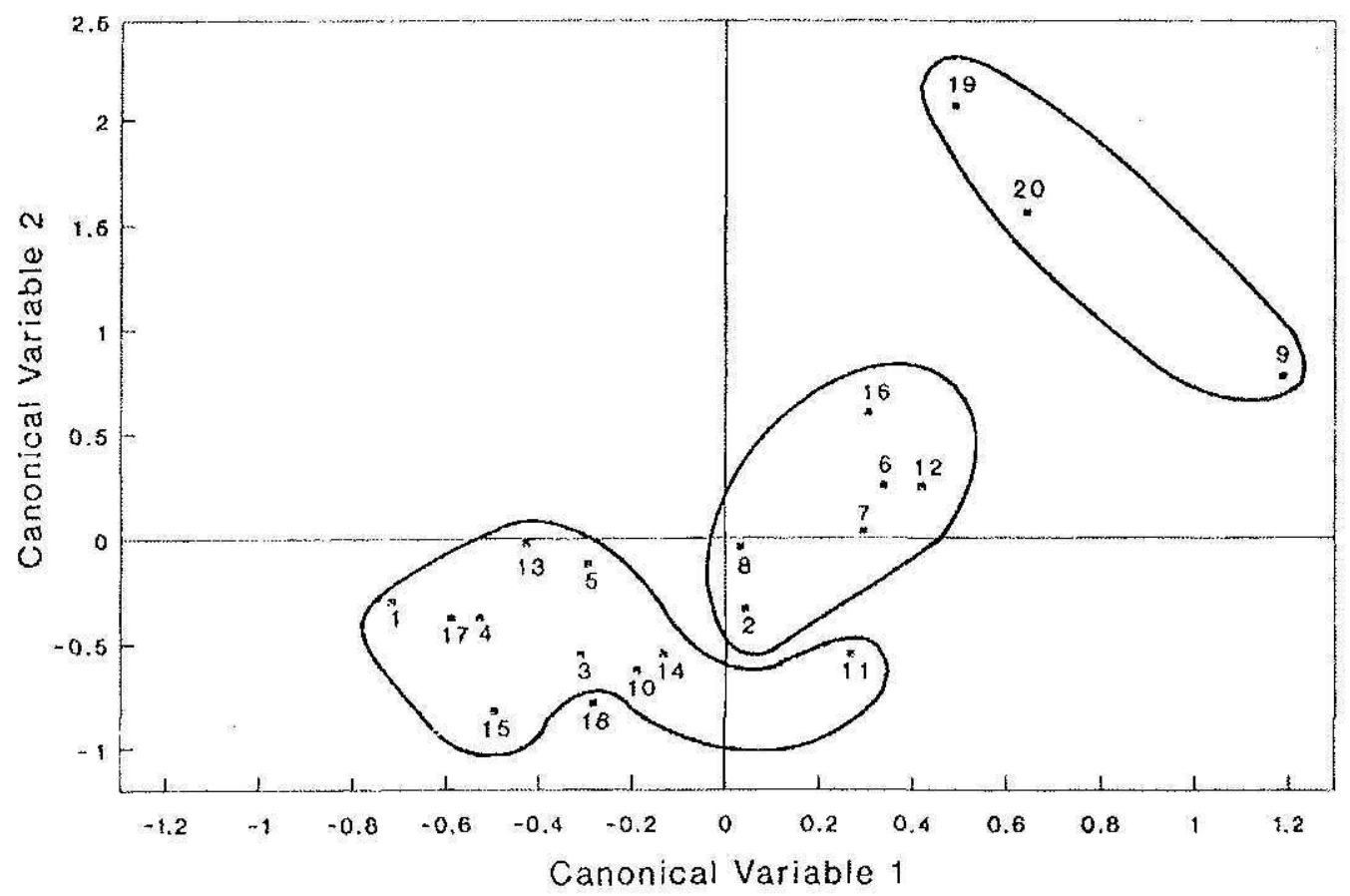

Ficure 2. Scatterplots of $J_{3}$ of 20 isolates of Heterorhabditis on the first and second canonical axes with three subgroups circled. Population 18 appears away from subgroup 2.

This species could not be identified by using existing keys, and was also confirmed as new by $G$. O. Poinar (personal communication). 
J. Agric. Univ. P.R. vOL. 79, NO. 1-2, JANUARY/APRIL 1995

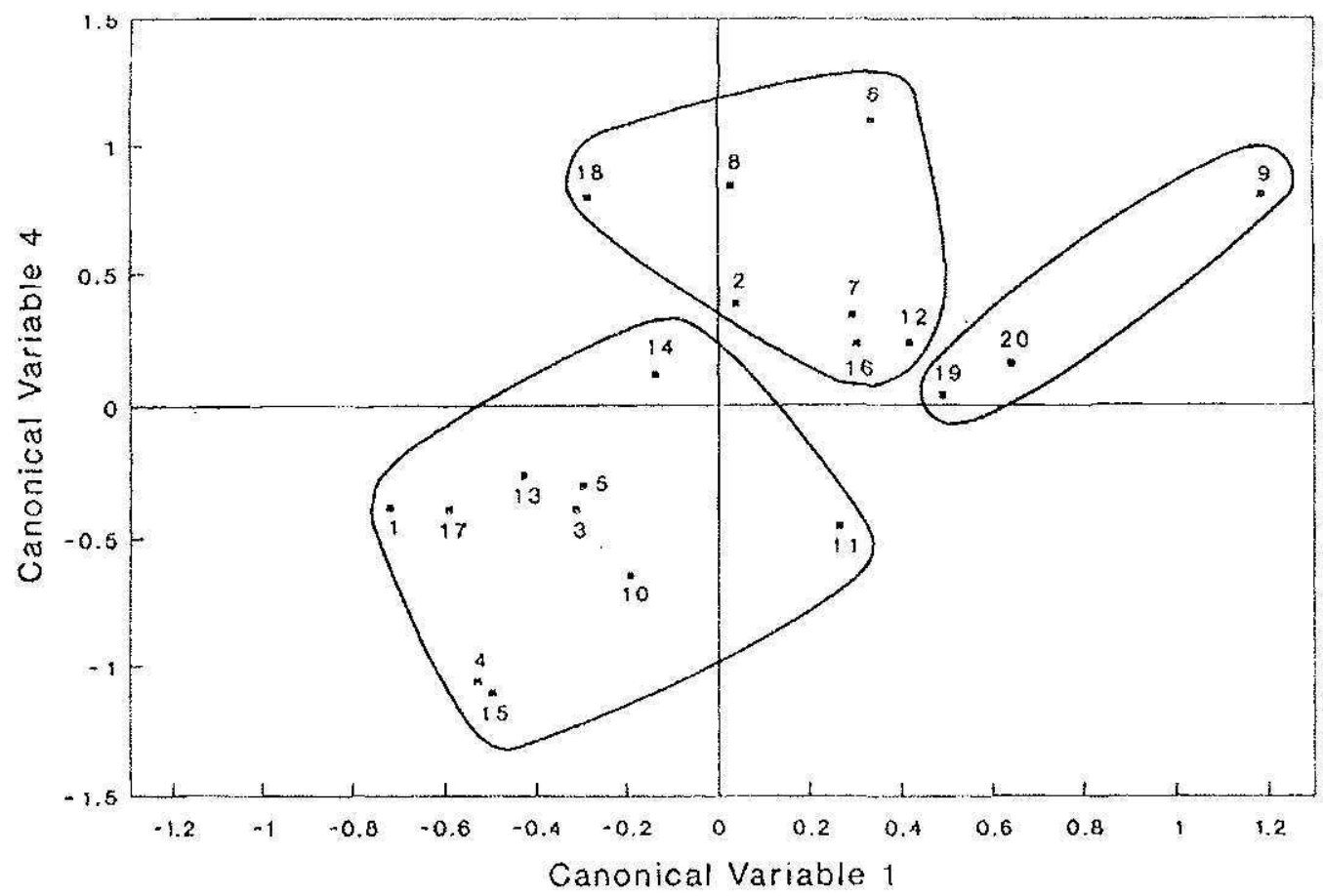

Frgure 3. Scatterplots of $\mathfrak{J}_{3}$ of 20 isolates of Heterorhabditis on the first and fourth canonical axes with three subgroups circled.

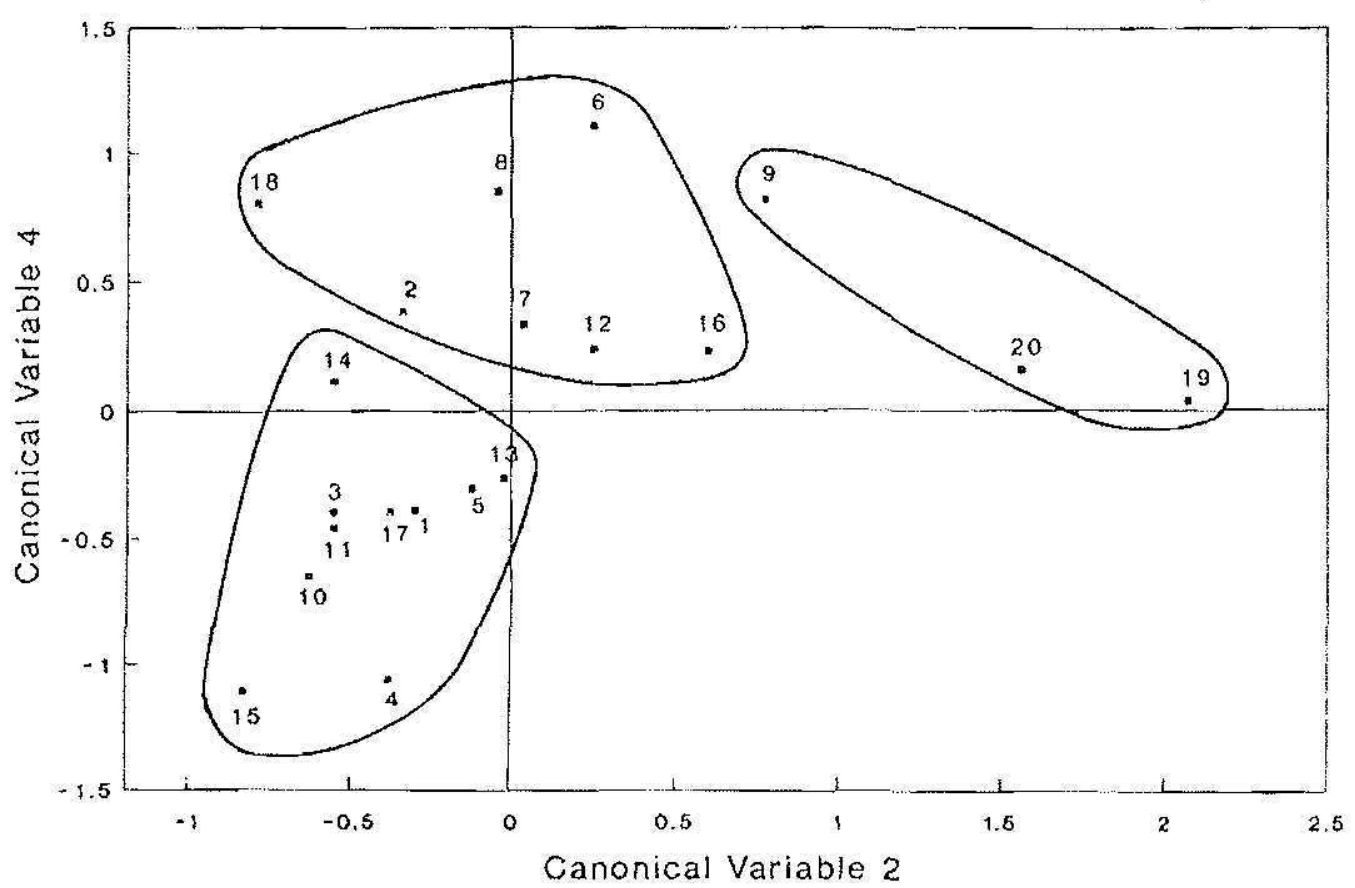

FIGURE 4. Scatterplots of $J_{3}$ of 20 isolates of Heterorhabditis on the second and fourth canonical axes with three subgroups circled. 


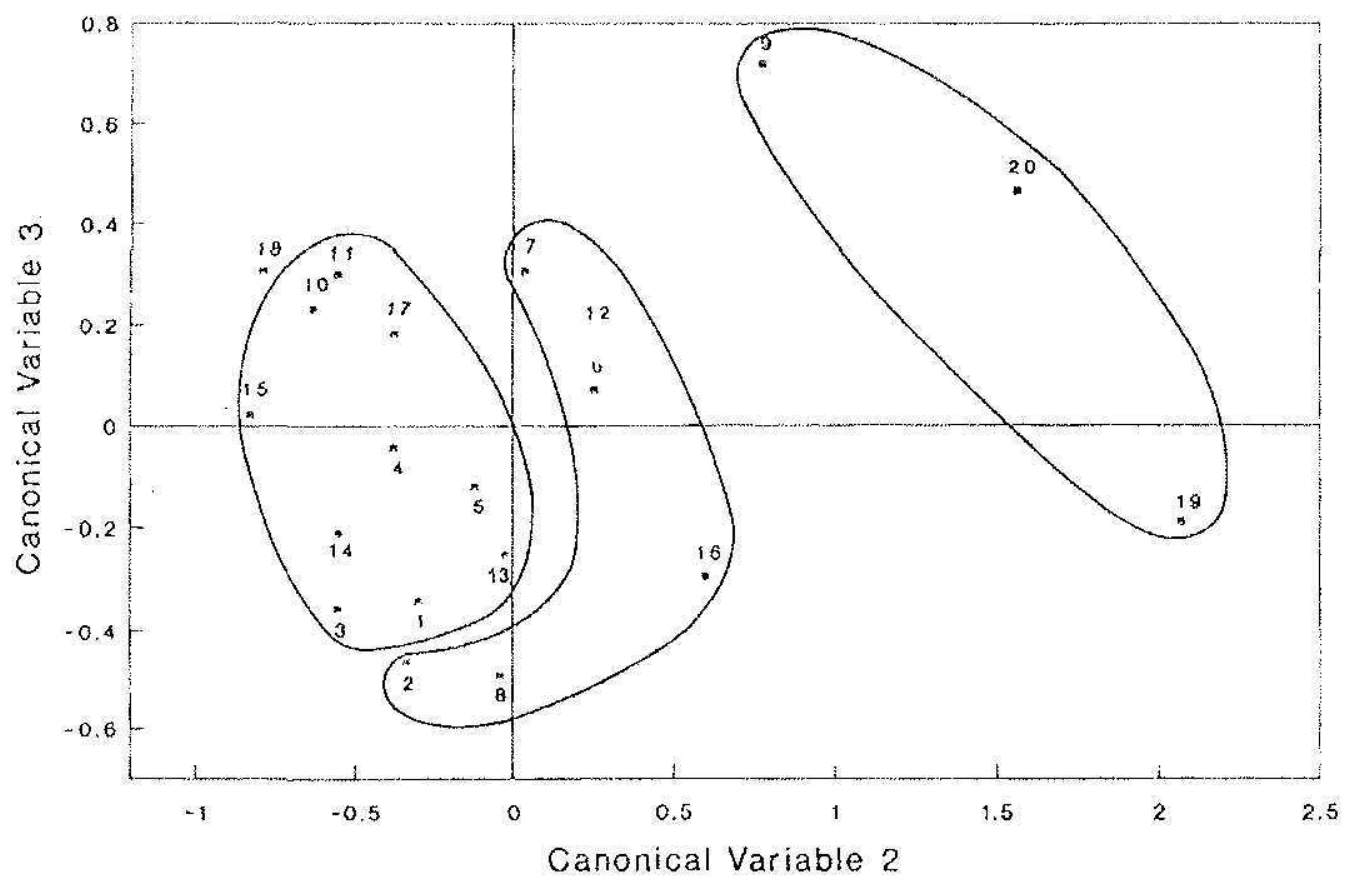

FliURe 5. ScatterpJots of $J_{3}$ of 20 isolates of Heterorhabditis on the second and third canonical axes with three subgroups circled. Population 18 appears away from subgroup 2.

the canonical analysis, could not be plotted in polygonals to demarcate the range of each isolate as Hirschmann and Rammah (1993) did for eight populations of Meloidogyne arenaria. Too much overlapping occurred among the various isolates of Heterorhabditis. Plotting polygonals using the four canonical variables selected (Table 2) was also confusing. We found that scatterplots with canonical means could best illustrate the relationship among the isolates. Scatterplots of canonical means have been previously used by other authors to separate populations of certain species (Cho and Robbins, 1991; Georgi, 1988; Lamberti and Ciancio, 1993).

Results of the HCA and the CDA were fairly consistent. The three subgroups delimited by HCA (Figure 1) were also observed in the scatterplots of the CDA (Figures 2 through 5) especially when borderlines were traced following the results obtained by the HCA. Subgroup 3 , composed of isolates 9,19 , and 20 , was the most consistent of the three. It appeared well demarcated by the HCA (Figure 1). Lamberti and Ciancio (1993) separated 49 populations belonging to the Xiphinema americanum group into five subgroups by HCA and Principal Component Analysis. Hirschmann and Rammah (1993) conducted a morphometric study of the females, males, and $\mathrm{J}_{2}$ of hypotriploid and triploid populations of $M$. arenaria with SDS, SDA, and CA. They dem- 
onstrated that in each life stage, the hypotriploid populations were set off to varying degrees from the triploid populations. In our study of Heterorhabditis, it appeared that subgroup 3 is distinctly demarcated in all four CDA scatterplots but more clearly so in two of them (Figures 2 and 5). Certain isolates, especially 11 and 18, were usually set off at the borderlines of subgroups 1 and 2, respectively, indicating relationship or dissimilarity with the three subgroups plotted (Figures 1 through 5). The location of the isolates on scatterplots depended on the contribution made by each canonical variable. Cho and Robbins (1991) separated three groups and four populations belonging to four different species from $23 X$. americanum sensu lato populations by using CDA scatterplots. Georgi (1988) employed CDA to investigate morphological variations in $X$. americanum and correlated them with regional differences. The latter study failed to separate mixed species clearly.

The information obtained in the present study indicates the existence of a new species of Heterorhabditis from Puerto Rico which appears closely related to $H$. bacteriophora and $H$. indicus. Our work also showed a large amount of variability among the isolates studied. It is difficult to determine at present whether or not the observed variability is an indication of the presence of other species. Studies underway on polyacrilamide gel electrophoresis should elucidate this problem.

\section{LITERATURE CITED}

1. Akhurst, R. J., 1987. Use of starch gel electrophoresis in the taxonomy of the genus Heterorhabditis (Nematoda: Heterorhabditidae). Nematologica 33:1-9.

2. Cho, M. R. and R. T. Robbins, 1991. Morphological variation among 23 Xiphinema americanum populations. J. Nematol. 23:134-144.

3. Curran, J., 1990. Molecular techniques in taxonomy. Pp. 63-74. In: R. Gaugler and H. R. Kaya, eds. Entomopathogenic Nematodes in Biological Control. CRS Press, Inc., Boca Ratón, Fl.

4. Figueroa, W., J. Román and M. A. Acosta, 1993. Isolates of entomogenous nematodes Heterorhabditis spp. and mortality of larvae of Galleria mellonella, Cylas formicarius, Euscepes postfasciatus and Cosmopolites sordidus. J. Agric. Univ. P.R. 77:5360.

5. Georgi, L. L., 1988. Morphological variation in Xiphinema spp. from New York ofchards. J. Nematol. 20:47-57.

6. Hirschmann, H. and A. Rammah, 1993. Morphometric evaluation of hypotriploid and triploid populations of Meloidogyne arenaria. J. Nematol, 25:121-135.

7. Huettle, R. N., D. W. Dickson and D. P. Kaplan, 1983. Biochemical identification of the two races of Radopholus similis by starch gel electrophoresis. J. Nematol. $15: 338-344$.

8. Hussey, R. S., 1979. Biochemical systematics of nematodes: A review. Helminth. Abs. Series B 48:141-148.

9. Khan, A., W. W. Brooks and H. Hirschmann, 1976. Cromonema heliothidis n. gen., $\mathrm{n}$. sp. (Steinernematidae:Nematoda), a parasite of Heliothidis zea (Noctuidae:Lepidoptera), and other insects. J. Nematol. 8:159-168. 
10. Lamberti, F. and A. Ciancio, 1993. Diversity of Xiphinema americanum group species and hierarchical cluster analysis of morphometrics. $J$. Nematol. 25:332-343.

11. Mota, M. M. and J. D. Eisenback, 1993. Morphometrics of Globodera tabacum tabacum, G. T. virginiae and G. T. solanacearum (Nematoda:Heteroderidael. $d$. Nematol. 25:148-160.

12. Norton, D. C. and P. N. Hinz, 1992. Morphometric variation and biogeography of Ogma menzeli and Criconema sphagni. J. Nematol. 24:399-403.

13. Pantone, D. J., J. A. Griesbach and A. R. Maggenti, 1987. Morphometric analysis of Anguina amsinckiae from three host species. J. Nematol. 19:158-163.

14. Poinar, G. O. Jr., 1992. Heterorhabditis indicus n. sp. (Rhabditida:Nematoda) from India: Separation of Heterorhabditis spp. by infective juveniles. Fund. Applied Nematol. 15:467-472.

15. Poinar, G. O. Jr, 1990. 'Taxonomy and biology of Steinernematidae and Heterorhabditidae. Pp. 23-61, In: R. Gaugler and H. K. Kaya, eds. Entomopathogenic Nematodes in Biological Control. CRS Press, Inc., Boca Ratón, Fl.

16. Poinar, C. O. Jr., 1979. Nematodes for Biolugical Control of Insects. CRS Press, Boca Ratón, Fl.

17. Poinar, G. O. Jr., 1975. Description and biology of a new insect parasitic rhabditoid, Heterorhabdutis bacteriophora n. gen., n. sp. (Rhabditida:Heterorhabditidae n. fam.), Nematologica 21:463-470.

18. Poinar, G. O. Jx, T. Jackson and M. Klein, 1987. Heterorhabditis megidis sp. n. (Heterorhabditidae:Rhabditida), parasitic of the Japanese beetle, Pompilia japonica (Scarabeidae:Coleoptera). Ohio. Proc. Helminth. Soc. Wash. 54:53-59.

19. Román, $J$ and H. Hirschmann, 1969. Morphology and morphometrics of six species of Pratylenchus. J. Nematol. 1:363-386.

20. SAS Institute Inc., 1982. SAS User's Guide: Statistics, 1982 ed. Cary, N.C., SAS Institute, Inc. 